CASE REPORT

\title{
Failure of adaptive servo-ventilation to correct central sleep disorder breathing in a patient with congestive heart failure
}

\author{
Ayush Gupta, J. C. Suri, Abhijeet Singh, M. K. Sen, S. Chakrabarti \\ Department of Pulmonary, Critical Care and Sleep medicine, VMMC and Safdariung Hospital, New Delhi, India.
}

DOI No: 10.5958/0974-0155.2015.00007.8

Indian J Sleep Med 2014; 9.4, 186-189

\begin{abstract}
We report a patient with congestive heart failure secondary to dilated cardiomyopathy who demonstrated central sleep apnea with Cheyne-Stokes breathing (CSA-CSB) on polysomnography. He was put on positive airway pressure therapy with adaptive servoventilation (ASV), which failed to correct the CSA-CSB condition. A backup rate was added with ASV, which eliminated apnea but periodic breathing persisted. Finally, enhanced expiratory rebreathing spacewas added and titrated with ASV to achieve normocapniausing transcutaneous $\mathrm{CO}_{2}$ monitoring that successfully stabilized the patient's breathing.
\end{abstract}

Keywords: Central sleep apnea, Cheyne-Stokes breathing,adaptive servoventilation.

\section{Introduction}

$\mathrm{T}$ The characteristic features of central sleep apnea (CSA) include an absence of drive to breathe during sleep, which leads to inadequate or noventilation and compromised gas exchange. Obstructive sleep apnea (OSA) shows continuing respiratory efforts, whereas CSA results in the absence of respiratory effort when the airflow stops. Similar to OSA, CSA poses several significant difficulties such as recurrent nighttime awakenings, extreme daytime sleepiness, and enhanced risk of adverse cardiovascular outcomes. CSA with Cheyne-Stokes breathing (CSACSB) is a form of periodic breathing, commonly observed in patients with congestive heart failure (CHF),

\section{Address for correspondence}

\section{Dr J.C. Suri}

Professor and Head

Department of Pulmonary, Critical Care and Sleep Medicine, VMMC and Safdariung Hospital

New Delhi, India

Email: docjcsuri@gmail.com in which central apneas alternate with hyperpnea that have a waxing-waning pattern of tidal volume and respiratory effort. ${ }^{1}$ In general, patients with CSA-CSB show hypocapnic condition, with a value of $\mathrm{PaCO}_{2}$ nearer than standard to the apneic threshold so that even a minor augmentation in ventilation pushes $\mathrm{PaCO}_{2}$ value below the threshold and initiates apnea. The chief negative effect of CSA-CSB on cardiovascular function seems to be extreme activity of the sympathetic nervous system caused by apnea-related hypoxia and awakenings from sleep. In addition to pharmacotherapy for CHF, nocturnal continuous positive airway pressure (CPAP), bilevel positive airway pressure (BiPAP), and ASV have been tried to treat CSA-CSB in sleep. ${ }^{2}$ We report a case of an elderly gentleman with CSA-CSB secondary to CHF who failed on nocturnal ASV.

\section{Case Report}

Our patient was a 75-year-old man, nonhypertensive, nondiabetic, heavy cigarette smoker with a history of 30 pack years, no history of alcohol intake, who presented 
to us with complaints of progressively increasing breathlessness for 2 years, orthopnea, cough with mucoid sputum, and pedal edema for 1 year. On examination, patient was in respiratory distress with a respiratory rate of $35 / \mathrm{min}$; pulse rate, $140 / \mathrm{min}$; blood pressure, $100 /$ $60 \mathrm{~mm} \mathrm{Hg}$; and saturation by pulse oximetry, $80 \%$ on room air. Patient showed jugular venous distension with bilateral pitting lower extremity edema. Chest auscultation revealed bilateral basal end inspiratory crepitations. Other system examination revealed no abnormal findings. All laboratory investigations were within normal limits except elevated NT-probrain natriuretic peptide levels $(17,332 \mathrm{pg} / \mathrm{dL})$. Arterial blood gas analysis done on room air showed a $\mathrm{pH}$ of 7.471, $\mathrm{PaO}_{2} 43 \mathrm{~mm}, \mathrm{PaCO}_{2} 31 \mathrm{~mm}$, and $\mathrm{HCO}_{3} 20 \mathrm{mmol}$. Chest skiagram revealed cardiomegaly with bilateral pleural effusion. ECG showed sinus tachycardia with no ST segment changes. Transthoracic $2 \mathrm{D}-\mathrm{ECHO}$ revealed left ventricular ejection fraction of $30 \%$ with global LV hypokinesia, mitral regurgitation, and all chambers dilated. Cardiac output measurement by arterial pulse wave contour analysis revealed a low cardiac output of $2.4 \mathrm{~L} / \mathrm{min}$ and systemic vascular resistance index of 3,350 dynes $/ \mathrm{s} / \mathrm{cm}^{-5}$. Coronary angiography was within normal limits. Patient was diagnosed to have CHF owing to dilated cardiomyopathy and was started on diuretics, angiotensin-converting enzyme inhibitors, antiplatelets, statins, and CPAP at $8 \mathrm{~cm} \mathrm{H}_{2} \mathrm{O}$ for pulmonary edema. However, patient was intolerant to CPAP with inability to sleep and was planned for polysomnography to look for reasons of CPAP failure. Diagnostic portion without PAP therapy revealed CSA-CSB with a CSA index of $48 / \mathrm{h}$ and marked sleep fragmentation with arousal index of $51 / \mathrm{h}$ (Figure 1 ). There was a significant desaturation with lowest $\mathrm{SpO}_{2}$ of $45 \%$. End tidal $\mathrm{CO}_{2}$ and transcutaneous $\mathrm{CO}_{2}\left(\mathrm{tcpCO}_{2}\right)$ monitoring (Radiometer, TCM 400, USA) were done, which showed wide fluctuations from 27 to $44 \mathrm{~mm} \mathrm{Hg}$ during CSA-CSB.

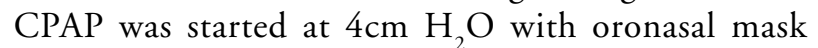
(Phillips Respironics;gel-based mask) and increased to $10 \mathrm{~cm}$ of $\mathrm{H}_{2} \mathrm{O}$ to eliminate CSA-CSB. However, central apneas persisted with periodic breathing, recurrent arousals, and desaturation. ASV (Auto ASV; Respironics, USA) was started using the same oronasal mask with initial settings of end expiratory positive airway pressure (EPAP) window of $4-8 \mathrm{~cm} \mathrm{H}_{2} \mathrm{O}$, and minimum and maximum pressure support of 0 and $20 \mathrm{~cm} \mathrm{H}_{2} \mathrm{O}$, respectively. We observed pressure variations in both EPAP and inspiratory PAP, consistent with activation of the device. CSAs persisted with hypocapnia $\left(\mathrm{tcpCO}_{2}=\right.$ $32 \mathrm{~mm} \mathrm{Hg}$ ) and periodic breathing (Figure 2). Addition of a backup rate of $22 / \mathrm{min}$ to ASV eliminated central apneas with an improvement in lowest $\mathrm{SpO}_{2}$ to $85 \%$ (Figure 3). However, CSB persisted with a further reduction in $\mathrm{tcpCO}_{2}$ to $20 \mathrm{~mm} \mathrm{Hg}$. In view of persistent periodic, unstable breathing with frequent arousals and hypocapnia, an enhanced expiratory rebreathing space (EERS) was added between the nonvented oronasal mask and the leak valve in the tubing to increase tcpCO $\mathrm{CO}_{2}$ to normocapnia (Figure 4). EERS was a corrugated tube used in the ventilatory circuit. The EERS was titrated from $5 \mathrm{~cm}(35 \mathrm{~mL})$ to $20 \mathrm{~cm}(140 \mathrm{~mL})$ in increments of 5 $\mathrm{cm}(35 \mathrm{~mL})$ with tcpCO $\mathrm{CO}_{2}$ monitoring to target levels of $40-45 \mathrm{~mm} \mathrm{Hg}$. Patient was put on ASV with EERS, and the periodic breathing stabilized with $\mathrm{tcpCO}$ increasing to $41-45 \mathrm{~mm} \mathrm{Hg}$ with no central apneas (Figure 5). The arousal index fell to $6 / \mathrm{h}$ and the lowest $\mathrm{SpO}_{2}$ improved to $91 \%$. The patient was successfully titrated with ASV with EERS using tcpCO $\mathrm{CO}_{2}$ monitoring.

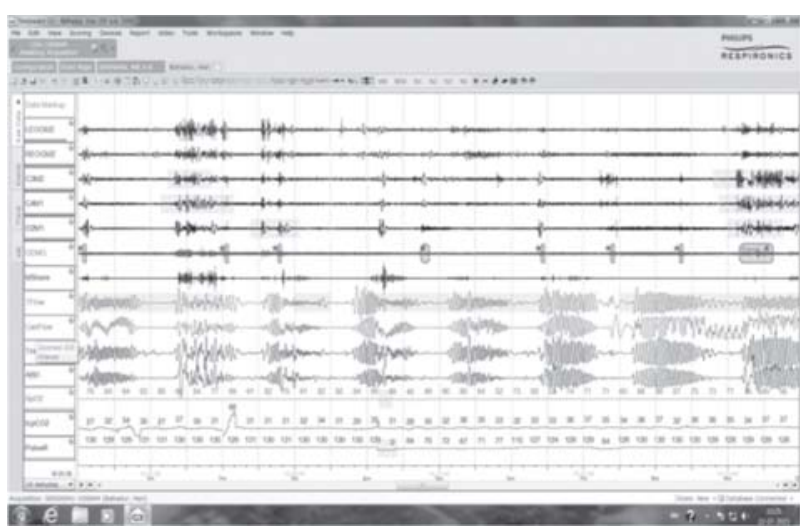

Figure 1: Cheyne-Stokes respiration with central sleep apnea in a patient with $\mathrm{CHF}$

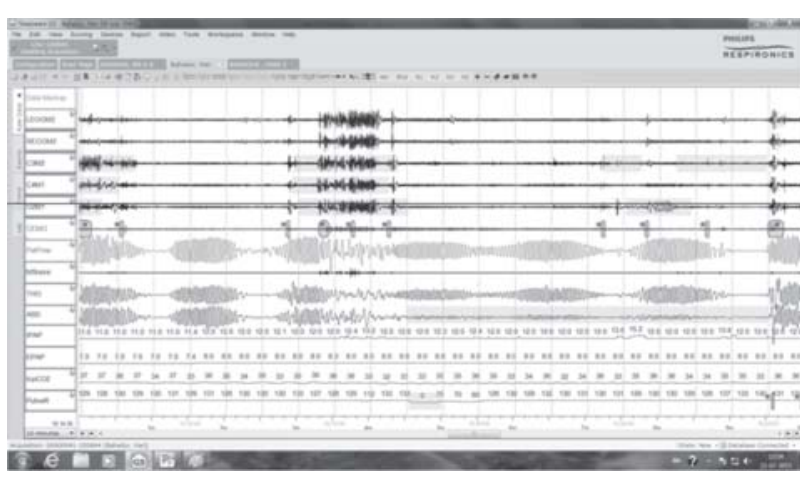

Figure 2: Adaptive servoventilation-CSA persistent with periodic breathing

Indian Journal of Sleep Medicine (IJSM), Vol. 9, No. 4, 2014 


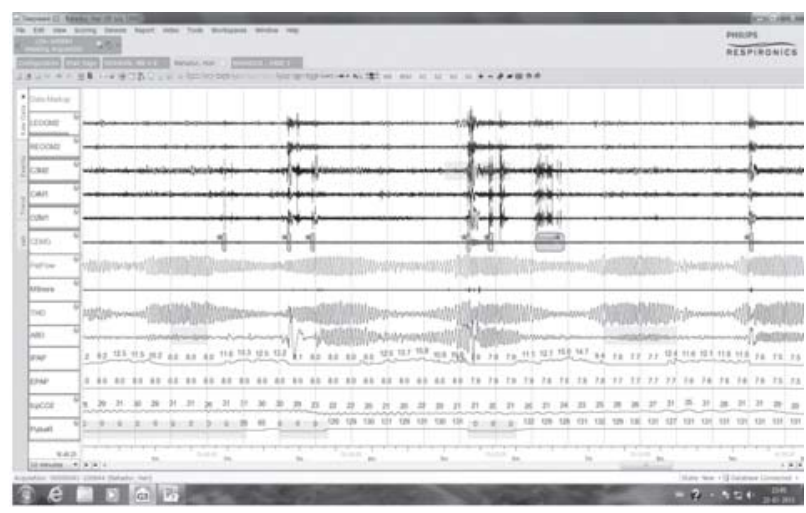

Figure 3: ASV with backup rate of 22/min-periodic breathing persisting on ASV with further hypocapnia

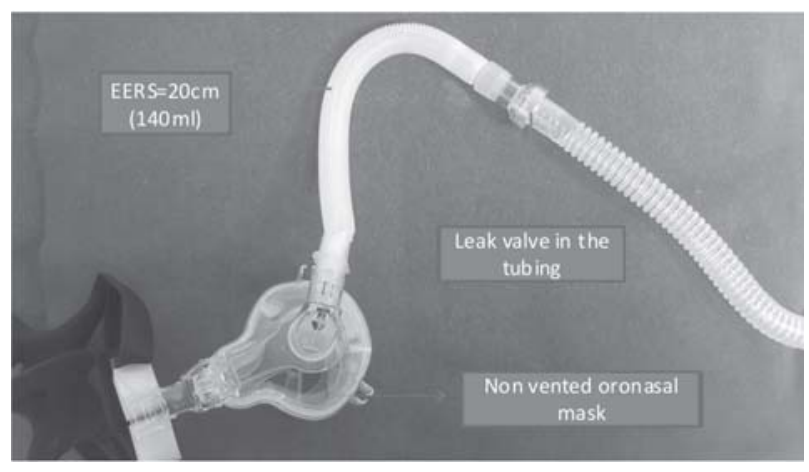

Figure 4: EERS added to the circuit

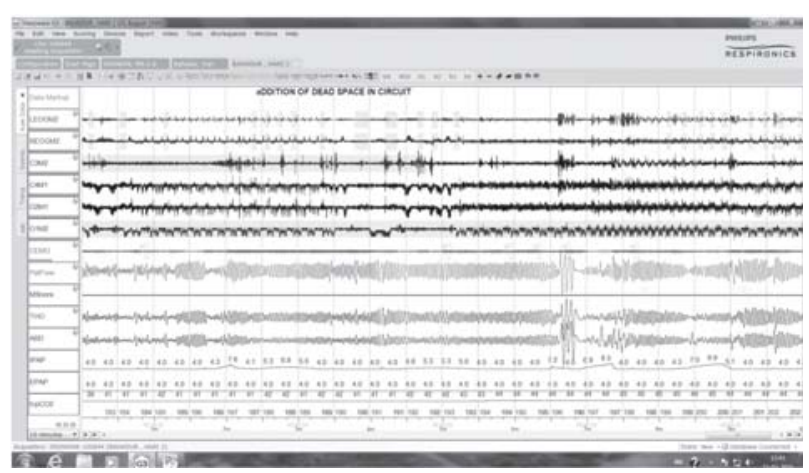

Figure 5: ASV with addition of enhanced expiratory rebreathing space (EERS)-tcpCO ${ }_{2}$ increased to $42-44$

\section{Discussion}

CSA-CSB in patients with heart failure arises because of respiratory control system instability. Ventilation depends on the chemical rather than the central respiratory control system during sleep, and the chief stimulation for ventilation while asleep is $\mathrm{PaCO}_{2}$. CSA during sleep happens when $\mathrm{PaCO}_{2}$ values go lower than the apnea threshold. A key factor predisposing to respiratory control system instability and CSA-CSB in $\mathrm{CHF}$ is chronic hyperventilation with eupneic $\mathrm{PaCO}_{2}$ close to the apnea threshold, a condition called as low $\mathrm{CO}_{2}$ reserve ${ }^{1}$. This chronic hyperventilation arises owing to the stimulation of pulmonary vagal irritant receptor by pulmonary congestion and elevations in the central and peripheral chemosensitivities. There are three major components of ventilatory control system-the plant gain, circulatory delay, and the controller gain-all of which are altered in patients with CSA-CSB. Plant gain is the change in $\mathrm{PaCO}_{2}$ for a given change in ventilation. Circulatory delay is the blood transit time from the lung to the peripheral and central chemoreceptors and is increased in patients with CHF. Prolongation of circulation time secondary to reduced cardiacoutput with delayed transmission of alteration in arterial blood gas tensions from the lung to the peripheral and central chemoreceptors contributes to the pathogenesis of CSACSB by facilitating ventilatory overshoot and undershoots. Controller gain is the change in ventilation for a given change in $\mathrm{PaCO}_{2}$. All the three together constitute the respiratory loop gain, which is a measure of respiratory stability, and a high loop gain indicates an inherently unstable system prone to frequent oscillation and inability to achieve a steady state ${ }^{1,3}$. Another important mechanism in the pathogenesis of CSA-CSB is repeated arousals. Although these arousals function as a protection mechanism in OSA tostopapneas and initiate pharyngeal muscles that enable recommencement of the airflow, in CSA, they initiate central apneas by aggravating ventilatory overshoot. The significant role of arousal in continuing ventilatory overshoot during periodic breathing is shown by the sturdy association between the magnitude of arousals and both ventilation during hyperpnea and consequent apnea period. Persistence of CSA-CSB in patients with CHF even after optimization of the pharmacotherapy predisposes them to repeated surges of sympathetic activity during sleep with adverse cardiovascular consequences and increased risk of premature death ${ }^{4}$. Never theless, controversy remains as to whether CSA-CSB is simply a reflectionof heart failure severity or whether it exerts independent adverse effects. Treatment options include CPAP, BiPAP, ASV, supplemental oxygen inhalation, $\mathrm{CO}_{2}$ inhalation, acetazolamide, lorazepam, and phrenic nerve stimulation $^{5-7}$. It is important to identify the exact pathophysiological mechanism responsible for CSA-CSB in a particular patient. Our patient showed persistent hypocapnia causing CSA-CSB even with CPAP and then ASV. The two methods to increase $\mathrm{CO}_{2}$ are to either 
reduce minute ventilation or to increase the dead space. The addition of EERS to ASV in our patient led to the restoration of normocapnia and stabilization of breathing. This technique of adding a dead space to the circuit is easier than artificial insufflation of $\mathrm{CO}_{2}$ gas in the circuit. ${ }^{8}$ End-tidal $\mathrm{CO}_{2}$ monitoring for EERS titration is not accurate with PAP therapy due to dilution of flows. The role of tcpCO $\mathrm{CO}_{2}$ monitoring for titration of EERS is not defined. We found it extremely useful to sequentially increase the amount of dead space till normocapnia was achieved and were successfully able to treat CSA-CSB, which had failed to correct on CPAP or ASV alone. The purpose of this case report is to highlight the fact that tcpCO ${ }_{2}$ monitoring should be an essential part of CPAP, BiPAP, or ASV titration for the management of CSACSB. It not only helps in understanding the exact mechanism of periodic breathing but also helps in titrating the exact amount of EERS to be added without doing repeated arterial blood gas analysis.

\section{References}

1. Yumino D, Bradley DT. Central sleep apnea and CheyneStokes respiration. Proc Am Thorac Soc.2008;5(2):226236.

2. Momomura S. Treatment of Cheyne-Stokes respirationcentral sleep apnea in patients with heart failure. $J$ Cardiol.2012;59(2):110-116.

3. Eckert DJ, Jordan AS, Merchia P, Malhotra A. Central sleep apnea: pathophysiology and treatment. Chest. 2007;131(2):595-607.

4. Koyama T, Watanabe H, Tamura Y, Oguma Y, Kosaka T, Ito $H$. Adaptive servo-ventilation therapy improves cardiac sympathetic nerve activity in patients with heart failure. Eur $J$ Heart Fail.2013;15(8):902-909.

5. Brown LK, Javaheri S. Adaptive servo-ventilation for the treatment of central sleep apnea in congestive heart failure: what have we learned?CurrOpinPulm Med.2014;20(6):550-557.

6. Reddy H, Dillard TA. A failure of adaptive servo-ventilation to correct central apneas in Cheyne-Stokes breathing. J Clin Sleep Med.2012;8(1):103-106.

7. Banno K, Okamura K, Kryger MH. Adaptive servoventilation in patients with idiopathic Cheyne-Stokes breathing. J Clin Sleep Med.2006;2(2):181-186.

8. Gilmartin G, McGeehan B, Vigneault K,et al. Treatment of positive airway pressure treatment-associated respiratory instability with enhanced expiratory rebreathing space (EERS). JClin Sleep Med.2010;6(6):529-538. 